\title{
Development of a Measure of Rehabilitation Adherence for Athletic Training
}

\section{Megan D. Granquist, Diane L. Gill, and Renee N. Appaneal}

\begin{abstract}
Context: Rehabilitation adherence is accepted as a critical component for attaining optimal outcomes. Poor adherence is recognized as a problem in the athletic training setting. Measurement has been inconsistent, and no measure has been developed for athletic training settings. Objective: To identify indicators of sportinjury rehabilitation adherence relevant to athletic training and develop a Rehabilitation Adherence Measure for Athletic Training (RAdMAT) based on these indicators. Design: Mixed methods, 3 steps. Setting: College athletic training facility. Participants: Practicing certified athletic trainers (ATCs; $n=7$ ) generated items, experts $(n=12)$ reviewed them, and practicing ATCs $(n=164)$ completed the RAdMAT for their most, average, and least adherent athlete. Main Outcome Measure: RAdMAT. Results: The RAdMAT is 16 items with 3 subscales. Subscales and total have good internal consistency and clearly discriminate among adherence levels. Conclusions: The RAdMAT is based on scholarly literature and clinical practice, making it particularly appropriate for use in athletic training clinical practice or for research purposes.
\end{abstract}

Keywords: compliance, sport injury, psychology, behavior

An estimated 380,000 student-athletes participate in the National Collegiate Athletic Association (NCAA) across 23 sports at more than 1000 institutions nationwide. ${ }^{1}$ The NCAA's Injury Surveillance System injury data from the 1988-89 to 2003-04 seasons across 15 sports have been summarized, indicating that during this 15-year span, 72,316 injuries were reported during games (an average of 13.79 injuries per 1000 athlete exposures), and 109,160 injuries were reported during practice (an average of 3.98 injuries per 1000 athlete exposures). ${ }^{2}$ Considering that an athlete must report the injury to medical staff and miss a practice or competition, and the medical staff must report the injury (via either paper or electronic form) to the Injury Surveillance System to be counted, these rates may be an underestimate of actual injury prevalence. Furthermore, it is logical to assume that many of the athletes with injuries required some degree of sport-injury rehabilitation to return to full function.

Granquist is with the Dept of Movement and Sports Science, University of La Verne, La Verne, CA. Gill and Appaneal are with the Dept of Kinesiology, University of North Carolina at Greensboro, Greensboro, NC. 
College athletes normally receive sport-injury rehabilitation services through their college or university's athletic training room, rather than a community-based sports medicine rehabilitation clinic (eg, physical therapy or physiotherapy). Rehabilitation adherence (eg, attendance, participation, exercise completion) is commonly accepted in sports medicine as a critical component for successful sport-injury rehabilitation outcomes. ${ }^{3-10}$ Poor rehabilitation adherence (eg, athlete working either too little or too much) may decrease overall rehabilitation outcomes (eg, functional ability, strength, range of motion $)^{11,12}$ and increase the chance of reinjury. ${ }^{3}$ Despite the ease of access for athletes to receive rehabilitation services, poor adherence is anecdotally reported as a common occurrence and recognized as a major problem affecting rehabilitation in the athletic training setting. ${ }^{6.13}$ Byerly et al ${ }^{14}$ reported that among 44 Division II college athletes, $63 \%$ were nonadherent in rehabilitation based on attendance and athletic trainers' ratings of athlete participation.

Outside the athletic training setting, sport-injury rehabilitation-adherence rates in community-based sports medicine rehabilitation settings (eg, physical therapy or physiotherapy clinics) range from $40 \%$ to $91 \% .^{12}$ In one study, physiotherapists reported that $55 \%$ of patients were not fully adherent with prescribed home modalities, and $51 \%$ of these patients were not fully adherent with prescribed rest from activity. ${ }^{9}$ Udry ${ }^{10}$ found adherence rates for athletes receiving community-based sports medicine rehabilitation after anterior cruciate ligament reconstruction to be $79 \%$, with highest adherence rates at the beginning of rehabilitation. Adherence rates below $100 \%$ pose a real problem in sport-injury rehabilitation in both athletic training and community-based sports medicine settings, and this research suggests that rehabilitation outcomes in these settings may be less than optimal.

Although the problem of poor adherence is widely recognized, the definition and measurement of rehabilitation adherence have been inconsistent. There is no clear conceptual model or definition of rehabilitation adherence, also termed compliance, ${ }^{4}$ in the sports medicine or sport psychology literature. A widely accepted definition of adherence among practitioners who use exercise as rehabilitation is "an active, voluntary collaborative involvement of the patient in a mutually acceptable course of behavior to produce a desired preventative or therapeutic result." 15, p20 Adherence in general medicine and health has been defined as "the degree to which patient behaviors coincide with the recommendations of healthcare providers." 6 ,p188s In line with these definitions, adherence in the sport-injury rehabilitation setting involves athletes' demonstrating behaviors that coincide with the recommendations of their health-care provider, who, in the athletic training setting, is the athletic trainer.

Given the lack of a consistently agreed-on definition of rehabilitation adherence, it is not surprising that measurement is problematic. There is no "gold standard" by which to rate adherence. Researchers have yet to systematically identify the behaviors that constitute rehabilitation adherence. In the athletic training setting, researchers have measured adherence using both attendance and athletic trainers' ratings of athlete participation, rehabilitation completion, following instructions/ advice, and receptivity to changes in rehabilitation. ${ }^{14,17}$ In community-based sports medicine rehabilitation clinics, adherence has been measured in a variety of ways via patient self-report and practitioner report, including patients' attendance, exercise/rehabilitation completion, following instructions/advice, receptivity to 
changes in rehabilitation, and compliance with home-care instructions and activity restrictions. ${ }^{5,9,11,18,19}$

To date, only 1 measure of rehabilitation adherence has been developed and published for use with sport injuries: the Sport Injury Rehabilitation Adherence Scale (SIRAS) ${ }^{20}$ The SIRAS was developed specifically for use in a communitybased sports medicine rehabilitation clinic. Although it has been shown to have good psychometric properties, ${ }^{20}$ it is arguably difficult to capture human behavior with 3 items. Because there is no gold-standard measure or agreed-on operational definition of rehabilitation adherence, it is also difficult to be sure that the SIRAS is really measuring adherence. In addition, rehabilitation in the college athletic training room may differ from rehabilitation at a community-based sports medicine rehabilitation clinic.

The current lack of a valid and reliable rehabilitation-adherence measurement tool specific to the athletic training setting is a noted hindrance in sport-injury rehabilitation-adherence research and clinical practice. ${ }^{21} \mathrm{~A}$ measure of adherence behaviors for rehabilitation must not only be useful in practice but also be conceptually based and psychometrically sound. Identifying and measuring the behaviors of sport-injury rehabilitation adherence in a college athletic training setting is vital to the further understanding and investigation of the overall rehabilitation process (eg, biopsychosocial factors that influence rehabilitation adherence, potential interventions to enhance adherence) and successful outcomes (eg, range of motion, strength, functional ability, future injury, etc). For a measure of rehabilitation adherence to be valid and useful, it must include athlete behaviors that are easily interpretable and observable by college athletic trainers, and rehabilitation-adherence behaviors must be generally agreed on by college athletic trainers who observe those behaviors.

The long-term goal of this research is to develop a survey measure that adequately assesses behaviors of rehabilitation adherence in an athletic training setting. For this project, rehabilitation adherence is operationally defined as behaviors an athlete demonstrates by pursuing a course of action that coincides with the recommendations of the athletic trainer. Although measure development and evaluation is a lengthy process that requires multiple investigations to establish reliability and validity, the purpose of this project was to develop a measure of rehabilitation adherence for athletic training that includes indicators of adherence identified by athletic trainers who are currently practicing in the college athletic training setting and that reflects the operational definition of rehabilitation adherence. It is expected that these indicators will include, but not be limited to, attendance and exercise completion and that the resulting Rehabilitation Adherence Measure for Athletic Training (RAdMAT) will reflect the operational definition, have acceptable psychometric properties, and be relevant for use in an athletic training setting.

\section{Methods}

The current study included 3 steps in the development of the RAdMAT. First, an initial survey of certified athletic trainers provided data to generate items. Second, an expert panel reviewed the preliminary items and provided feedback pertaining to their content and clarity. Finally, in step 3, the preliminary measure was administered to a larger sample of certified athletic trainers to provide data for initial reliability and validity analyses. 


\section{Step 1: Item Generation}

For the initial step of item generation, certified athletic trainers who were active in clinical practice completed an open-ended online survey to generate a list of rehabilitation-adherence behaviors.

Participants. The measure is intended for use by practitioners, and clinical relevance is of primary importance, so all participants were certified athletic trainers employed full-time to work with college athletes and were directly involved in conducting or supervising rehabilitation for college athletes in the United States. From a list of recommended potential participants, the investigator chose participants who were representative of college certified athletic trainers (ie, Division I, II, III, NAIA; male/female; sports coverage; area of the country). ${ }^{22}$

Sixteen certified athletic trainers were contacted, and 7 completed the survey. After the 7 responses were analyzed we determined that saturation had been reached and further responses would likely yield no new information. The 7 participants ( 3 men, 4 women) who completed the step 1 initial survey came from across the country (ie, CA, FL, MS, TX, WI, WA) and represented all NCAA divisions, as well as NJCAA Division I. All participants were certified athletic trainers, had master's degrees, and had practiced athletic training in the college setting for an average of 11.3 years (range 4-30 y). Participants collectively had athletic training coverage responsibilities for nearly all sports and were directly involved in conducting or supervising rehabilitation for college athletes.

Measure. The step 1 survey included basic demographic information and provided participants with the definition of rehabilitation adherence used in this investigation: behaviors an athlete demonstrates by pursuing a course of action that coincides with the recommendations of the athletic trainer. The main items asked participants to define rehabilitation adherence, to list behaviors that indicate rehabilitation adherence and nonadherence, and to list anything else they use to determine whether an athlete is adherent or nonadherent. Participants were also asked to think about athletes they had worked with who had good and poor adherence and to list behaviors that indicated their good or poor adherence. Questions were open ended, and space was provided for participant comments.

Procedures. After obtaining institutional-review-board approval, the primary investigator contacted potential participants via e-mail with a letter explaining the purpose of the study and asking for their participation. The 7 athletic trainers who agreed to participate clicked on an online survey link embedded in the recruitment e-mail. Survey Monkey, an Internet-based survey tool, was used to administer surveys and collect responses. Participants were again provided with information on the purpose of the study, and informed consent was obtained before beginning the survey. Participants were asked to complete the survey within 1 week. After the 7 responses were analyzed it was determined that saturation (ie, the same responses continually appeared and no new responses were given) had been reached and further responses would likely yield no new information. Therefore, no additional participants were recruited.

Analysis. Responses were independently analyzed by the investigator and a second coder using methods described by Mason. ${ }^{22}$ The investigator identified 
all key words and phrases to compile a list of adherence behaviors. From that list of behaviors, items that were similar or related were grouped into categories. The second coder (a doctoral student and certified athletic trainer) independently followed the same procedures. Discrepancies were resolved through discussion.

The list was then distributed back to participants via e-mail to check for accuracy and further additions. No further additions or revisions were made, and the draft version of the RAdMAT was created for expert review in stcp 2.

\section{Step 2: Expert Review}

The behaviors identified from step 1 were organized into a survey format and sent via e-mail to a different set of experts for input on item content, clarity, and format. Expert ratings were used to revise the draft measure and provide an initial assessment of content validity.

Participants. As in step 1, the investigator obtained recommendations from practicing certified athletic trainers working with college athletes and also included nonpracticing experts who had expertise in athletic training education, sport psychology, or rehabilitation research. Participants were considered experts if they had published multiple peer-reviewed articles in their respective fields.

Out of 18 potential participants contacted, 12 (6 men, 6 women) reviewed the draft measure. Three participants were faculty or researchers in sport psychology, 3 had dual appointments (ie, faculty and athletic training), and 6 were practicing athletic trainers. Ten participants were from the United States (GA, MA, MS, NC, OH, OR, UT, and WA), 1 was from the United Kingdom, and 1 was from Australia. The US participants represented all NCAA Divisions.

Measure. The instructions included the definition of rehabilitation adherence and a list of the 49 preliminary items (behaviors of adherence). The experts rated the content (Does the behavior accurately measure rehabilitation adherence?) and clarity (Is the item clear and understandable?) of each item on a 3-point scale (yes, maybe, no) and were allowed the opportunity to reword items or make additions or clarifications to better represent adherence.

Procedures. As in step 1, the investigator contacted experts via e-mail to explain the project and obtain informed consent. Experts completed demographic information, rated items for content and clarity, and were also asked for formatting suggestions. As in step 1, the survey was distributed and data were collected via Survey Monkey.

Analysis. Expert ratings and comments were compiled, and items that were not rated as having appropriate content or being clear by all experts were reviewed and revised or deleted based on the ratings and comments. The investigator reviewed the results and revisions with a second reviewer (a faculty member with experience in development of psychological measures). after agreement on all revisions, the 26 revised items were sent back to the experts, who were asked whether items needed further revision to verify accuracy and for additional comments. The preliminary 25 -item RAdMAT was created from these final expert revisions. 


\section{Step 3: Athletic Trainer Survey}

The preliminary 25-item RAdMAT was sent via e-mail to certified athletic trainers, who completed the measure based on athletes they had worked with in rehabilitation over the past year. Specifically, participants were asked to rate the most adherent, least adherent, and a typical athlete they had worked with.

Participants. Eligible participants included 1000 certified athletic trainers who were members of the National Athletic Trainers' Association (NATA) and had given the NATA permission to be contacted via e-mail by the NATA national office. Participants' member type was either "regular certified" or "student certified;" and they were self-identified as working in a college or junior college in the United States.

Of the 1000 e-mails that were sent from the NATA national office, at least 46 e-mails were not delivered. Of the remaining 954 valid emails, 198 potential participants started the online survey, and 164 of those completed the survey for the most adherent athlete. Among the 164 participants ( 79 men, 85 women), 45 were head athletic trainers, 81 were assistant athletic trainers, 18 were athletic trainers, 5 were directors of athletic training education programs, 1 was a clinical coordinator of an athletic training education program, 13 were faculty, and $1 \mathrm{did}$ not report a job title. Participants had been certified for an average of 10.4 years (range 1-38 y) and had worked in the college setting for an average of 9.3 years (range 1-41 y). All NCAA athletics divisions, as well as NJCAA and club sports, were represented. All NCAA sports were represented, as well as rugby, crew, cheerleading, dance, equestrian, rodeo, squash, judo, badminton, synchronized swimming, and skating.

Measures. After providing demographic information participants completed the preliminary 25-item RAdMAT that was developed through steps 1 and 2. Instructions included the definition of rehabilitation adherence, and then participants were asked to consider 3 athletes (most adherent, least adherent, average) with whom they had worked and who had completed rehabilitation in the past year. Respondents rated each of those 3 athletes using the preliminary RAdMAT; each item was rated on a 4 -point scale $(1=$ never, $4=$ always $)$. Possible scores ranged from 25 to 100, with higher scores indicating greater adherence.

In addition to the RAdMAT, the SIRAS was also administered. The SIRAS consists of 3 items that ask the athletic trainer to rate athletes on their intensity of rehabilitation completion, frequency of following practitioner instructions and advice, and receptivity to changes in rehabilitation on a 5-point scale. The 3 items are summed to obtain a total SIRAS score that ranges from 3 to 15 , with higher scores indicating greater adherence.

Procedures. The investigator contacted the NATA and received permission to use the member-contact service to collect data. NATA members were contacted through this NATA service and were provided a cover letter (as per NATA contact-list-use agreement) providing a brief overview of the purpose of the study and a link to the survey. E-mails were sent by the NATA national office to a random sample of 1000 participants who met the participation criteria. Participants were provided with information on the purpose of the study, and informed consent was obtained before they began the survey. On the survey, 
participants were asked to complete the RAdMAT and the SIRAS 3 times; that is, they were asked to rate 3 different athletes' rehabilitation adherence (most adherent athlete, least adherent athlete, average adherent athlete that had completed rehabilitation in the last year) using both measures. Athlete order (most adherent and least adherent) was counterbalanced so that half the participants rated their most adherent first and the other half rated their least adherent first. Survey Monkey was used to distribute surveys and collect responses. A followup message was sent 2 weeks after the initial e-mail from the national office in an attempt to get additional responses.

Analyses. SPSS version 16 was the statistical package used for analyses. Descriptives, internal consistency reliability analyses, multivariate analysis of variance (MANOVA), analysis of variance (ANOVA) and exploratory factor analyses were conducted. For this investigation there were 164 participants and 25 initial items, a ratio of 6.56:1 participants to items. Although this small number of participants may limit exploratory factor analyses, Costello and Osborne ${ }^{23}$ suggest that this ratio is acceptable.

\section{Results}

\section{Step 1: Item Generation}

In the first step of item generation, the investigator classified responses into 3 general categories: presence (attendance, etc), active participation (follows instructions, etc) and positive attitude (gives 100\% effort, etc), and the second coder independently classified them into 7 categories: attendance, timeliness, effort, attitude, personal attributes/motivation, communication, and restrictions/instructions (follows prescribed plan, etc). The investigator and second coder then reviewed the results and reached agreement on combined and ignored items, which resulted in 3 broad categories with 10 subcategories. A list of adherence behaviors, including all categories identified and agreed on by both coders, was redistributed to participants for an accuracy check. No further modifications were made, and the 49-item draft version of the RAdMAT was created (Appendix 1).

\section{Step 2: Expert Review}

Based on the ratings and review of the 49 items, 23 items were deleted because they were not consistently rated as clear and accurate or were repetitious of better items. From the remaining items, 14 were revised based on experts' comments, which resulted in 26 items.

Three additional revisions were made based on the review of the modified items: Question 5 ( 2 on preliminary measure) was changed from shows up to arrives, question 27 (12) added communicates with athletic trainer, and in question 31 (15) has a positive outlook was deleted. This resulted in the 25-item preliminary RAdMAT (Appendix 2), which consists of 3 categories and 10 subcategories as described in step 1, with 2 items related to attendance (items 1 and 2), 12 items related to active participation (items 3-14), and 11 items related to positive attitude/ effort (items 15-25). 


\section{Step 3: Athletic Trainer Survey}

Internal Consistency. Internal consistency analyses were run for the RAdMAT and SIRAS for the most-, least-, and average-adherent data using the raw data. The 25-item RAdMAT demonstrated high internal consistency for the most-, average-, and least-adherent-athlete data (Cronbach's $\alpha=.927, .944$, and .926, respectively). The 3 -item SIRAS demonstrated moderate internal consistency for the most-, average-, and least-adherent-athlete data (Cronbach's $\alpha=.591, .861$, .771 , respectively).

Item Discrimination: Comparisons of Most-, Least-, and Average-AdherentAthlete Data. Total scores for the RAdMAT (25 item) and SIRAS were calculated for the most adherent, least adherent, and average adherent athlete. Missing items for the RAdMAT were replaced with the series mean if no more than 2 items were missing on a scale, so scale totals were calculated on 164 participants for the most-adherent-athlete data, 159 participants for the least-adherent-athlete data, and 145 participants for the average-adherent-athlete data. Means for all scale items and totals fell as expected; the most-adherent-athlete data had the highest means, the least-adherent-athlete data had the lowest means, and the averageadherent-athlete data fell between the 2 .

Within-subject MANOVAs were conducted to compare the 3 athlete groups (ie, most-, least-, and average-adherent athletes) on the RAdMAT items to determine whether all individual items adequately discriminate among adherence levels. Separate within-subject ANOVAs were conducted for each of the RAdMAT items to examine item discrimination. All RAdMAT items significantly discriminated among the 3 adherence levels; the univariate $F$ value for the adherence effect was statistically significant $(P<.001)$ for all 25 items, with all $F$ values over 87 . In addition, follow-up simple contrasts revealed that all 3 levels differed significantly $(P<.001)$ for each item, with all $F$ values over 55 . The univariate $F$ value for the adherence effect was also statistically significant $(P<.001)$ for the 3 SIRAS items.

Factor Structure of the RAdMAT. Principal-component factor analyses with varimax rotation were used to explore the factor structure of the most-adherentathlete raw data. First, the factor analysis was allowed to run with unlimited factors, which yielded a 6-factor solution. Based on the scree plot and percentage of variance explained, either a 2-factor or a 3-factor solution seemed to best fit the data and also fit with the conceptual model of steps 1 and 2 . Factor 1 in the 6-factor solution includes attendance and several of the attitude/effort items, and factor 2 includes attendance and several participation items. Factors 3 and 4 include a few items related to attitude and communication. The 3 items loading on factor 5 (completes exercises correctly, reports pain, and works well on own) do not reflect a particular category, do not load on any other stronger factors, and, as a result, may not be necessary. The 2 items related to home-based exercise loaded on factor 6 , which accounted for little variance. Furthermore, home-based exercise is not easily observed or evaluated by athletic trainers, so these items have limited clinical use. In addition, items that did not load over .50 on any factor do not seem to add any useful information.

Additional results of factor analyses with forced 3-factor, 2-factor, and 1-factor solutions were similar to those of the 6-factor solution, suggesting 3 factors, with 
items suggested as being dropped with the 6-factor solution still not providing additional information. Thus, those items $(5,7,8,9,10,14,17,23$, and 24) were dropped and the remaining 16 items run in a factor analysis, which resulted in a 3 -factor solution that explains $57.93 \%$ of the variance and seems to be the best fit. Factor 1 contains attitude/effort items (15, 16, 18-22, and 25), factor 2 contains attendance/participation items (1-4 and 6), and factor 3 contains communication items (11-13; Table 1). A forced 1-factor solution showed that all items strongly loaded on a single factor. The suggested 3-factor and 1-factor solutions also fit well for the least- and average-adherent-athlete data.

Based on results of the current study, the suggested 16-item RAdMAT (Appendix 3) has 3 subscales (attendance/participation, communication, and

Table 1 Factor Matrix for the Rehabilitation Adherence Measure for Athletic Training (16 Items)

\begin{tabular}{|c|c|c|c|}
\hline \multirow[b]{2}{*}{ Item } & \multicolumn{3}{|c|}{ Components } \\
\hline & 1 & 2 & 3 \\
\hline 1. attends scheduled rehabilitation sessions & .140 & .708 & .104 \\
\hline 2. arrives at rehabilitation on time & .452 & .494 & -.129 \\
\hline $\begin{array}{l}\text { 3. follows the athletic trainer's instructions during reha- } \\
\text { bilitation sessions }\end{array}$ & .325 & .649 & .019 \\
\hline 4. follows the prescribed rehabilitation plan & .070 & .729 & .178 \\
\hline 6. completes all tasks assigned by the athletic trainer & .181 & .747 & .127 \\
\hline 11. asks questions about his/her rehabilitation & .136 & -.020 & .747 \\
\hline $\begin{array}{l}\text { 12. communicates with the athletic trainer if there is a } \\
\text { problem with the exercises }\end{array}$ & .308 & .127 & .792 \\
\hline $\begin{array}{l}\text { 13. provides the athletic trainer feedback about the reha- } \\
\text { bilitation program }\end{array}$ & .131 & .221 & .786 \\
\hline 15. has a positive attitude during rehabilitation sessions & .792 & .077 & .266 \\
\hline 16. has a positive attitude toward the rehabilitation process & .692 & .011 & .336 \\
\hline 18. gives $100 \%$ effort in rehabilitation sessions & .712 & .323 & .100 \\
\hline 19. is self-motivated in rehabilitation sessions & .640 & .400 & .095 \\
\hline 20. is an active participant in the rehabilitation process & .580 & .378 & .315 \\
\hline 21. stays focused while doing rehabilitation exercises & .604 & .106 & .030 \\
\hline 22. is motivated to complete rehabilitation & .575 & .353 & .313 \\
\hline 25. shows interest in the rehabilitation process & .586 & .291 & .376 \\
\hline Initial eigenvalues & 6.36 & 1.71 & 1.20 \\
\hline$\%$ of variance & 39.72 & 10.71 & 7.50 \\
\hline Cumulative \% variance & 39.72 & 50.44 & 57.93 \\
\hline
\end{tabular}


attitude/effort) and could also be used as a single factor representative of adherence. Internal consistencies for the most-, average-, and least-adherent-athlete data for the subscales and total are acceptable (Table 2). Correlational analyses revealed significant, positive, and strong relationships between the 16-item RAdMAT total and SIRAS total. Relationships between the RAdMAT subscales and SIRAS total were also all significant, positive, and strong (Table 3 ).

Table 2 Scale and Subscale Descriptives for the Rehabilitation Adherence Measure for Athletic Training (16 Items)

\begin{tabular}{lccccc}
\hline & Mean & Min & Max & SD & Cronbach's $\alpha$ \\
\hline Attendance-most adherent & 18.79 & 14 & 20 & 1.52 & .767 \\
Attendance-average adherent & 15.38 & 9 & 20 & 2.01 & .764 \\
Attendance-least adherent & 11.17 & 5 & 18 & 2.61 & .824 \\
Communication-most adherent & 10.47 & 6 & 12 & 1.57 & .759 \\
Communication-average adherent & 8.65 & 4 & 12 & 1.67 & .796 \\
Communication-least adherent & 6.22 & 3 & 11 & 1.97 & .748 \\
Attitude-most adherent & 25.03 & 15 & 28 & 2.80 & .872 \\
Attitude-average adherent & 22.99 & 14 & 32 & 3.80 & .910 \\
Attitude-least adherent & 15.24 & 8 & 27 & 3.80 & .884 \\
Total-most adherent & 54.28 & 37 & 60 & 4.85 & .890 \\
Total-average adherent & 47.03 & 32 & 63 & 6.45 & .919 \\
Total-least adherent & 32.63 & 16 & 50 & 7.00 & .901 \\
\hline
\end{tabular}

The attendance/participation subscale range is $5-20$. The communication subscale range is $3-12$. The attitude/effort subscale range is $8-32$. The total scale range is $16-64$.

Table 3 Correlation Matrix for the Rehabilitation Adherence Measure for Athletic Training (RAdMAT) and Sport Injury Rehabilitation Adherence Scale (SIRAS)

\begin{tabular}{lccccc}
\hline & $\mathbf{1}$ & $\mathbf{2}$ & $\mathbf{3}$ & $\mathbf{4}$ & $\mathbf{5}$ \\
\hline 1. Total RAdMAT (16-item) & - & $.933^{*}$ & $.865^{*}$ & $.962^{*}$ & $.898^{*}$ \\
2. Subscale: attendance/participation & & - & $.746^{*}$ & $.837^{*}$ & $.868^{*}$ \\
3. Subscale: communication & & & - & $.762^{*}$ & $.744^{*}$ \\
4. Subscale: attitude/effort & & & & - & $.858^{*}$ \\
5. Total SIRAS & & & & & - \\
\hline
\end{tabular}

$* P<.01$. 
Comparisons of Totals and Subscales for Most-, Least-, and Average-AdherentAthlete Data. Within-subject MANOVAs comparing the total scores for the most-, least-, and average-adherent-athlete data discriminate among adherence levels: $F_{2,163}=614.77, P<.001, \eta^{2}=.790$. Follow-up simple contrasts comparing level 1 (most) versus 3 (average) and 2 (least) versus 3 (average) showed that all 3 levels differed significantly from each other: $F_{2,163}=173.72, P<.001, \eta^{2}=.516$, and $F_{2.163}=392.18, P<.001, \eta^{2}=.755$, respectively.

Furthermore, within-subject MANOVAs comparing the 3 subscale scores for the most-, least-, and average-adherent-athlete data also discriminate among adherence levels: multivariate $F_{6.158}=211.19, P<.001, \eta^{2}=.889$. Univariate tests were significant for all 3 subscales: attendance, $F_{2,326}=645.73, P<.001, \eta^{2}=.798$; communication, $F_{2,326}=299.07, P<.001, \eta^{2}=.647$; and attitude, $F_{2,326}=395.22, P$ $<.001, \eta^{2}=.708$. Follow-up simple contrasts also showed that all 3 levels differed significantly from each other. For the attendance/participation subscale, contrasts comparing level 1 (most) versus 3 (average) and 2 (least) versus 3 (average) revealed strong effects: $F_{1,163}=370.75, P<.001, \eta^{2}=.695$, and $F_{1,163}=392.18, P<.001, \eta^{2}$ $=.706$, respectively. For the communication subscale, contrasts comparing level 1 versus 3 and 2 versus 3 revealed moderate effects: $F_{1,163}=139.91, P<.001, \eta^{2}=$ .462 , and $F_{1,163}=194.18, P<.001, \eta^{2}=.544$, respectively. For the attitude/effort subscale, contrasts comparing level 1 versus 3 and 2 versus 3 revealed weak and strong effects: $F_{1,163}=36.82, P<.001, \eta^{2}=.184$, and $F_{1,163}=400.24, P<.001, \eta^{2}$ $=.711$, respectively.

MANOVA comparing the 3 SIRAS scores also revealed significant differences across adherence levels, $F_{2,328}=23.10, P<.001, \eta^{2}=.124$, and the contrasts comparing levels 1 versus $3, F_{1,164}=45.32, P<.001, \eta^{2}=.217$, and 2 versus $3, F_{1.164}=$ 9.09. $P<.003, \eta^{2}=.053$, were statistically significant. However, as the eta-square values indicate, differences on the SIRAS scores were not as strong as differences on the RAdMAT scores.

Relationships between adherence level and the 16-item RAdMAT total and subscales were all positive, significant, and moderately strong $(r \geq .704, P<.01)$.

\section{Discussion}

The purpose of this project was to identify indicators that reflect the operational definition of rehabilitation adherence (behaviors an athlete demonstrates by pursuing a course of action that coincides with the recommendations of the athletic trainer) and are relevant to a college athletic training setting and to develop a rehabilitation adherence measure for athletic training (RAdMAT) based on these indicators.

As demonstrated with the results of step 1, athletic trainers identified behaviors that were generally consistent with previous literature on rehabilitation adherence, with items relating to attendance, following instructions, and completing exercises. ${ }^{5,9,18-20,24}$ The current investigation is unique, however, in capturing these behaviors in an athletic training setting. Because of this, several responses were novel and add to our understanding of rehabilitation-adherence behaviors in the athletic training setting.

Novel responses include timeliness (ie, punctual to rehabilitation sessions) and communication. Previous studies used attendance as a measure of rehabilitation 
adherence $e^{5,10,14,19,20,24}$ but did not include timeliness. As is demonstrated in all steps of this project, athletic trainers consider timeliness an important behavior in judging rehabilitation adherence, and timeliness is clearly different from attendance. Two previous studies addressed communication in the athletic training room as an important factor for rehabilitation adherence, ${ }^{6,25}$ but the literature does not identify communication as a rehabilitation-adherence behavior.

From these sample responses, it is clear that athletic trainers likely use other cues (eg, athlete personality, behaviors occurring outside of the athletic training room, etc) to judge adherence. It is important to keep this in mind when interpreting the scores of the RAdMAT or any measure of adherence. To address this, a measure of adherence should include rehabilitation-adherence behaviors that are easily observable in the athletic training setting; the RAdMAT meets this standard.

The preliminary 25-item RAdMAT fit the conceptual model and reflected the behaviors identified in steps 1 and 2 . It also demonstrated good internal consistency and discriminated among varying levels of adherence. However, several items were redundant, and factor analyses provided an improved 16-item version.

Both a 3-factor solution and a 1-factor solution fit the final items for the most-, average-, and least-adherent-athlete data, suggesting that the RAdMAT may be used with 3 subscales (attendance/participation, communication, and attitude/ effort), as well as with a total score. The final 16-item RAdMAT includes 5 attendance/participation items, 3 communication items, and 8 attitude/effort items. Internal consistencies for these subscales and the totals for the most-, average-, and least-adherent-athlete data are all high (Cronbach's $\alpha>.748$ ), indicating that items contribute to the subscale and the total. as demonstrated with MANOVA and follow-up contrasts, each subscale clearly differentiates between the most, average, and least adherent athlete, indicating that both the subscales and totals may be used in future research to differentiate adherence levels. Although further analyses may refine the items and measure, internal consistencies and item discrimination are acceptable for the 16-item RAdMAT.

We expected the RAdMAT to be as psychometrically sound as and more relevant to an athletic training setting than the 3-item SIRAS that was developed for use in the community-based sports medicine setting. The results of this investigation suggest that it is at least as psychometrically sound as the SIRAS, and both the RAdMAT and SIRAS total scores significantly differentiate adherence groups. The RAdMAT appears to have a slight advantage over the SIRAS in detecting between-groups differences in adherence levels. The RAdMAT, with 3 subscales (attendance/participation, communication, and attitude/effort), can be used to examine relationships of these 3 factors to rehabilitation-adherence antecedents and outcomes. Although previous literature has endorsed the SIRAS as valid and reliable in the community-based sports medicine setting and appropriate for measuring athletes' adherence in a specific rehabilitation session, results from the current investigation suggest that the RAdMAT may be more appropriate for measuring athletes' adherence behaviors across sessions during rehabilitation in the athletic training setting.

Given the high correlation between the total scores on the RAdMAT and SIRAS ( $r=.898, P<.01$ ), the 3 -item SIRAS may be preferred as a quick, singlesession measure or for repeated assessments over several sessions. However, the 3 subscales of the RAdMAT are a distinct advantage with only a slightly longer 
measure. For example, an athlete may attend all of his or her rehabilitation sessions in a timely manner and complete all required exercises but may display poor effort, thus decreasing the effectiveness of the exercises. The RAdMAT could identify that profile, and the athlete's effort could be the focus of an intervention to increase overall rehabilitation adherence.

\section{Strengths and Limitations}

The use of certified athletic trainers who were currently practicing in the college setting to generate items is a strength because the measure is intended for use by practitioners and clinical usefulness is of primary importance. This sets the RAdMAT apart from the SIRAS and helps ensure that the RAdMAT is reflective of adherence behaviors specific to the athletic training setting and is relevant to athletic trainers.

Although every attempt was made to ensure that the sample in step 1 for item generation was representative (university division, area of the country, etc), this small selective sample may limit generalizability. Collected responses were independently analyzed by the investigator and a second coder. The use of a second coder and participant check confirms the accuracy of the compiled adherence behaviors and supports the content validity of the items on the RAdMAT measure. The RAdMAT covers a broader array of adherence behaviors than the SIRAS. The clinical and research experts further supported the content validity of the measure items.

The participant response rate was low in step 3. It is unknown how many potential participants actually received the recruiting e-mail, and several factors may have contributed to this low rate. Potential participants may not have received the group message from NATA because their e-mail servers may have filtered the recruiting e-mail as spam. Potential participants were asked to complete the online survey within 2 weeks at the end of June, when potential participants may not be checking their e-mail. Despite the low response rate, participants represented all NCAA athletics divisions, as well as NJCAA and club sports, and all NCAA sports, as well as several other activities.

Data from the sample of athletic trainers provided good support for the internal consistency of the measure and its ability to discriminate among adherence levels. However, knowing the outcome of rehabilitation in this retrospective study design may have altered the athletic trainers' perception of rehabilitation behaviors. The use of recall cases is a limitation, but the measure discriminated among these recalled cases, providing initial validity evidence that can be further tested with actual cases in prospective designs.

\section{Future Research With the RAdMAT}

Follow-up investigations should continue to examine the factor structure and psychometric properties of the RAdMAT. First, a follow-up investigation similar to step 3 might be conducted with a larger sample to further examine the psychometric properties of the RAdMAT. Although the current sample was sufficient for development of the preliminary measure, larger samples are needed to confirm the factor structure and internal consistency of the scale and subscales, as well as correlations 
with SIRAS and ability to discriminate among adherence levels. To improve on the retrospective design, athletic trainers could complete the RAdMAT, SIRAS, and a separate overall adherence rating for athletes during their rehabilitation using a prospective design. Adherence might be measured repeatedly (ie, weekly) during rehabilitation until the athlete is fully cleared to return to play. Repeated measures and completion by multiple raters could be used to establish test-retest and interrater reliability of the RAdMAT.

Although the measure was developed specifically for use in an athletic training setting, the individual items on the measure are not context specific. Items are specific to rehabilitation behavior, regardless of whether it occurs in an athletic training setting. Therefore, the RAdMAT may also be a useful clinical and research tool in a variety of rehabilitation settings other than athletic training. Future research may investigate the use of the RAdMAT in sport-injury rehabilitation settings other than athletic training.

Currently, there is no empirical evidence that supports the mediation role of adherence between rehabilitation antecedents and outcomes. Based on the results of this study, the RAdMAT has sufficient reliability and validity to justify its use in research examining these predictors and outcomes of rehabilitation adherence. Although the SIRAS may be a valid measure of rehabilitation adherence, the subscales of the RAdMAT provide more information regarding adherence behaviors. For example, the RAdMAT may be useful in examining relationships in the Brewer et $\mathrm{al}^{11}$ model of hypothesized relationships among psychological factors, rehabilitation adherence, and rehabilitation outcome that are widely assumed but seldom tested. Further studies will likely move beyond examining relationships to investigate strategies and interventions aimed at improving outcomes (eg, functional ability, quality of life, etc).

\section{Conclusions}

Previous literature shows that adherence is a problem and suggests that poor adherence affects rehabilitation in the athletic training setting. ${ }^{13,14}$ The current lack of a valid and reliable rehabilitation-adherence measurement tool specific to the athletic training setting is a noted hindrance in sport-injury rehabilitationadherence research and clinical practice. ${ }^{21}$ Identifying and measuring sport-injury rehabilitation-adherence behaviors in a college athletic training setting is vital to the further understanding and investigation of the overall rehabilitation process (eg, biopsychosocial factors that influence rehabilitation adherence, potential interventions to enhance adherence) and successful outcomes (eg, range of motion, strength, functional ability, future injury).Although there is no gold standard for adherence measures, the current results indicate that the RAdMAT is at least as good as the SIRAS for measuring rehabilitation-adherence behaviors in the athletic training setting. The RAdMAT has the advantage of including subscales that assess more than simply attending rehabilitation and completing the recommended exercises. These subscales may be useful for guiding practice and interventions to enhance sport-injury rehabilitation adherence. The RAdMAT differentiates between the most, least, and average adherent athletes, providing evidence for its validity. Furthermore, total scores and subscales for the most, least, and average adherent athletes were significantly related to the SIRAS, which has been found to be a reliable measure 
of adherence in the community-based sports medicine setting. Both the SIRAS and RAdMAT discriminated among the most-, least-, and average-adherent-athlete cases, but the RAdMAT was slightly better at group discrimination, as indicated by larger eta-square values. Additional testing of both measures is needed to determine whether one is more appropriate than the other for the athletic training setting.

In conclusion, the RAdMAT was based on a clear definition, focused on behaviors, and underwent multiple revisions and reviews by coders, as well as field experts. The development steps involved having practicing athletic trainers identify and confirm relevant adherence behaviors. Thus, the RAdMAT has both a conceptual base and a base in clinical athletic training practice, making it particularly relevant to and appropriate for athletic training settings. With only 16 items in a simple format, the RAdMAT is also relatively easy to use, whether by athletic trainers in clinical practice or for research purposes.

\section{Acknowledgement}

We thank Sandra J. Shultz, PhD, ATC, and Beverly J. Levine, $\mathrm{PhD}$, for their service on the doctoral dissertation committee from which this manuscript was derived. We also thank the National Athletic Trainers' Association for the use of their member database for participant recruitment, the sport psychology and athletic training experts who assisted with survey development, and the athletic trainers who participated in the study.

\section{References}

1. Jones S, Levine S. 2006-07 Guide for the College-Bound Student-Athlete. Iowa City, IA: National Collegiate Athletic Association; 2006.

2. Hootman JM, Dick R, Agel J. Epidemiology of collegiate injuries for 15 sports: summary and recommendations for injury prevention initiatives. $J$ Athl Train. 2007;42(2):311319.

3. Arnheim DD, Prentice WE. Principles of Athletic Training. 10th ed. Boston: McGraw Hill; 2000.

4. Bassett SF. The assessment of patient adherence to physiotherapy rehabilitation. New Zealand Journal of Physiotherapy. 2003;31(2):60-66.

5. Bassett SF, Prapavessis H. Home-based physical therapy intervention with adherenceenhancing strategies versus clinic-based management for patients with ankle sprains. Phys Ther. 2007;87:1132-1143.

6. Fisher AC, Mullins SA, Frye PA. Athletic trainers' attitudes and judgments of injured athletes' rehabilitation adherence. J Athl Train. 1993;28(1):43-47.

7. Flint F. Psychology of Sport Injury: A Professional Achievement Self-Study Program Course. Champaign, IL: Human Kinetics; 1998.

8. Kolt GS, Brewer BW, Pizzari T, Schoo AMM, Garrett N. The sport injury rehabilitation adherence scale: a reliable scale for use in clinical physiotherapy. Physiotherapy. 2007;93:17-22.

9. Taylor AH, May S. Threat and coping appraisal as determinants of compliance to sports injury rehabilitation: an application of protection motivation theory. I Sports Sci. 1996;14:471-482.

10. Udry E. Coping and social support among injured athletes following surgery. J Sport Exerc Psychol. 1997;19:71-90.

11. Brewer BW, Van Raalte JL, Cornelius AE, et al. Psychological factors, rehabilitation adherence, and rehabilitation outcome after anterior cruciate ligament reconstruction. Rehabil Psychol. 2000;45(1):20-37. 
12. Brewer BW. Adherence to sport injury rehabilitation programs. J Appl Sport Psychol. 1998;10:78-82.

13. Fisher AC, Scriber KC, Matheny ML, Alderman MH, Bitting LA. Enhancing athletic injury rehabilitation adherence. J Athl Train. 1993;28(4):312-318.

14. Byerly PN, Worrell T, Gahimer J, Domholdt E. Rehabilitation compliance in an athletic training environment. J Athl Train. 1994;29(4):352-355.

15. Meichenbaum D, Turk DC. Facilitating Treatment Adherence: A Practitioner's Guidebook. New York: Plenum; 1987.

16. Vitolins MZ, Rand CS, Rapp SR, Ribisl PM, Sevick MA. Measuring adherence to behavioral and medical interventions. Control Clin Trials. 2000;21:188S-194S.

17. Albinson CB, Petrie TA. Cognitive appraisals, stress, and coping: preinjury and postinjury factors influencing psychological adjustment to sport injury. J Sport Rehabil. 2003;12:306-322.

18. Brewer BW, Cornelius AE, Van Raalte JL, et al. Age-related differences in predictors of adherence to rehabilitation after anterior cruciate ligament reconstruction. $J$ Athl Train. 2003;38(2):158-162.

19. Scherzer CB, Brewer BW, Cornelius AE, et al. Psychological skills and adherence to rehabilitation after reconstruction of the anterior cruciate ligament. $J$ Sport Rehabil. 2001;10:165-172.

20. Brewer BW, Van Raalte JL, Petitpas AJ, et al. Preliminary psychometric evaluation of a measure of adherence to clinic-based sport injury rehabilitation. Phys Ther Sport. 2000;1(3):68-74.

21. Levy AR, Polman RC, Clough PJ, McNaughton LR. Adherence to sport injury rehabilitation programmes: a conceptual review. Res Sports Med. 2006;14:149-162.

22. Mason J. Qualitative Researching. 2nd ed. Thousand Oaks, CA: Sage; 2002.

23. Costello $\mathrm{AB}$, Osborne JW. Best practices in exploratory factor analysis: four recommendations for getting the most from your analysis. Pract Assess Res Eval. 2005;10(7):1-9.

24. Brewer BW, Cornelius AE, Van Raalte JL, et al. Protection motivation theory and adherence to sport injury rehabilitation revisited. Sport Psychologist. 2003;17:95-103.

25. Fisher AC, Hoisington LL. Injured athletes' attitudes and judgments toward rehabilitation adherence. J Athl Train. 1993;28(1):48-50.

\section{Appendix 1: 49-Item Draft Version of the RAdMAT}

\section{Presence}

a. Attendance

1. attends rehabilitation regularly

2. attends all rehabilitation sessions

b. Timeliness

3. is punctual to rehabilitation sessions

4. is timely to rehabilitation sessions

5. shows up to rehabilitation on time

6. allows enough time to complete rehabilitation program

\section{Active Participation}

a. Follows instructions

7. obeys instructions

8. follows the athletic trainer's recommendations 
9. follows the prescribed rehabilitation plan

b. Complies with activity restriction

10. complies with restrictions

11. limits things he or she not supposed to do

c. Completes exercises in athletic training room

12. completes all tasks assigned by the athletic trainer

13. completes exercises

14. completes entire rehabilitation protocol

15. completes all tasks

16. correctly performs exercises

17. consistently performs exercises

18. complies with directed exercises

d. Complies with home regimens

19. completes assigned home exercises

20. completes any "homework" that is assigned

21. completes home rehabilitation program

22. completes home modalities

e. Communicates with athletic trainer

23. has good communication

24. asks questions

25. gives suggestions

26. communicates with the athletic trainer

27. communicates if there is a problem with the exercises

28. communicates effectiveness of rehabilitation prescription

29. provides feedback

30. knows when to report pain or discomfort

\section{Positive Attitude}

a. Positive attitude

31. has a positive outlook

32. has a positive attitude toward rehabilitation

33. has a positive attitude toward the athletic trainer

34. good attitude toward the rehabilitation process

35. is enthusiastic

36. is energetic

37. has positive self-talk

38. is pleasant to work with

b. Effort

39. gives $100 \%$ effort 
40. demonstrates effort

41. has a strong work ethic

42. takes initiative to help himself or herself

43. is an active participant in the rehabilitation process

44. concentrates on exercises

c. Motivation

45. is motivated

46. is prepared for rehabilitation sessions

47. works well on his or her own

48. is independent

49. shows interest in rehabilitation process

\section{Appendix 2: Preliminary 25-item RAdMAT}

Please rate the athlete on each item using the scale: $1=$ never, 2 = occasionally, 3 =often, 4 = always.

1. Attends scheduled rehabilitation sessions

2. Arrives at rehabilitation on time

3. Follows the athletic trainer's instructions during rehabilitation sessions

4. Follows the prescribed rehabilitation plan

5. Complies with physical activity restrictions

6. Completes all tasks assigned by the athletic trainer

7. Completes exercises correctly in rehabilitation sessions

8. Completes assigned home exercises

9. Completes home rehabilitation modalities (ie, ice, heat, etc)

10. Communicates well with the athletic trainer

11. Asks questions about his or her rehabilitation

12. Communicates with the athletic trainer if there is a problem with the exercises

13. Provides the athletic trainer feedback about the rehabilitation program

14. Reports pain or discomfort when appropriate

15. Has a positive attitude during rehabilitation sessions

16. Has a positive attitude toward the rehabilitation process

17. Is easy to work with in rehabilitation

18. Gives $100 \%$ effort in rehabilitation sessions

19. Is self-motivated in rehabilitation sessions

20. Is an active participant in the rehabilitation process

21. Stays focused while doing rehabilitation exercises

22. Is motivated to complete rehabilitation

23. Is prepared for rehabilitation sessions 
24. Works well on his or her own during rehabilitation sessions

25. Shows interest in the rehabilitation process

\section{Appendix 3: Final Rehabilitation Adherence Measure for Athletic Training (RAdMAT)}

Please rate the athlete on each item using the scale: $1=$ never, 2 =occasionally, 3 = often, 4 = always.

1. Attends scheduled rehabilitation sessions

2. Arrives at rehabilitation on time

3. Follows the athletic trainer's instructions during rehabilitation sessions

4. Follows the prescribed rehabilitation plan

5. Completes all tasks assigned by the athletic trainer

6. Asks questions about his or her rehabilitation

7. Communicates with the athletic trainer if there is a problem with the exercises

8. Provides the athletic trainer feedback about the rehabilitation program

9. Has a positive attitude during rehabilitation sessions

10. Has a positive attitude toward the rehabilitation process

11. Gives $100 \%$ effort in rehabilitation sessions

12. Is self-motivated in rehabilitation sessions

13. Is an active participant in the rehabilitation process

14. Stays focused while doing rehabilitation exercises

15. Is motivated to complete rehabilitation

16. Shows interest in the rehabilitation process 